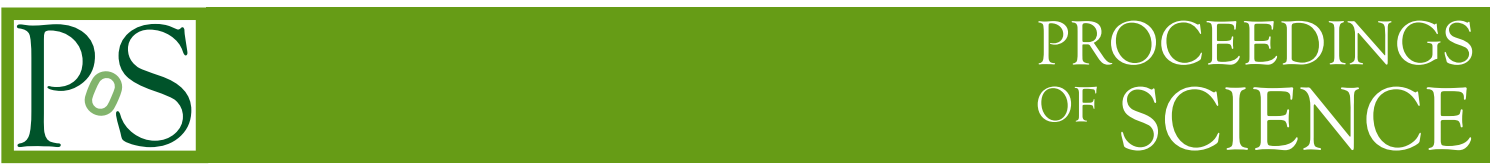

\title{
The operation and performance of the TOP detector at the Belle II experiment
}

\author{
Kazuki Kojima*on behalf of the Belle II TOP group \\ Nagoya University, \\ Furo-cho, Chikusa-ku, Nagoya, Aichi 464-8602, Japan \\ E-mail: kojima@hepl.phys.nagoya-u.ac.jp
}

The SuperKEKB/Belle II experiment, the successor of the KEKB/Belle experiment at KEK, started the physics data taking with the full detector system in March 2019. The Time-of-Propagation (TOP) detector was integrated into the Belle II detector for charged particle identification in the barrel region. The TOP detector mainly consists of quartz radiators, Micro-Channel-Plate (MCP) PMTs, and front-end electronics. It reconstructs a ring image of Cherenkov photons generated by an incident particle. It measures the timing of each detected photon with an accuracy of less than 100 ps for good $K / \pi$ separation.

In the operation of the TOP detector, harsh beam-induced background in the high luminosity environment is one of the critical issues to keep the particle identification performance. We developed various tools to monitor MCP-PMT performance and to identify and fix errors arising from front-end electronics during data taking. The TOP detector provides $85 \% \mathrm{~K}$ identification efficiency at a $10 \% \pi$ misidentification rate in the data at the early stage of the experiment. In these proceedings, we present the operation status and the performance by the summer of 2021 .

\footnotetext{
*** The European Physical Society Conference on High Energy Physics (EPS-HEP2021), ***

*** 26-30 July $2021 * * *$

*** Online conference, jointly organized by Universität Hamburg and the research center DESY ***
}

\footnotetext{
${ }^{*}$ Speaker
} 


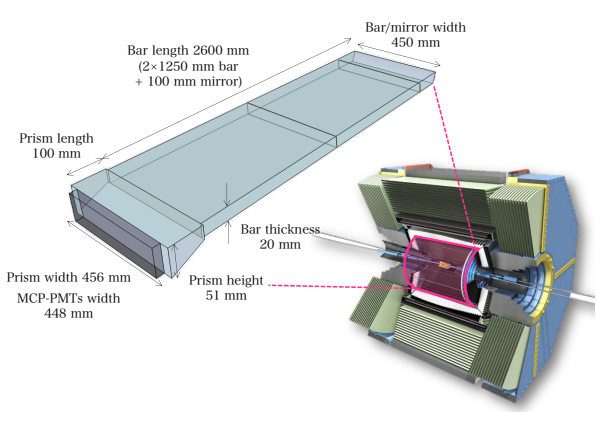

(a)

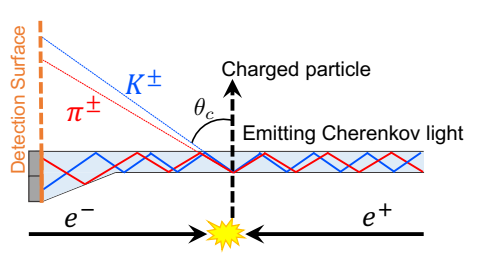

(b)

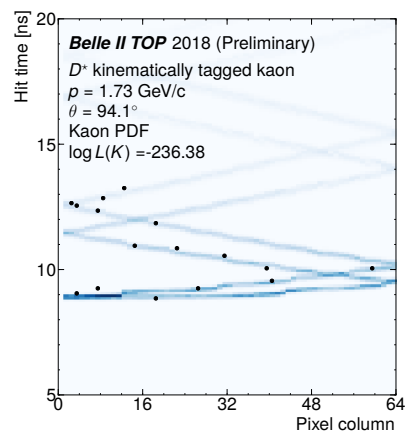

(c)

Figure 1: (a) A schematic view of a module of the TOP detector in the barrel region at the Belle II detector. (b) Cherenkov light paths from a charged particle passing through the TOP module. (c) Photosensor pixel column versus time of photon hits from $K$ in data (black points) over the expected probability density function for the $K$ hypothesis (blue bands).

\section{Introduction}

The SuperKEKB/Belle II experiment is an upgraded experiment of the former KEKB/Belle experiment with an electron-positron collider at the energy of the $\Upsilon(4 S)$ resonance at the High Energy Accelerator Research Organization (KEK) to achieve unprecedented integrated luminosity of $50 \mathrm{ab}^{-1}$ and instantaneous luminosity of $6.5 \times 10^{35} \mathrm{~cm}^{-2} \mathrm{~s}^{-1}$. The largest dataset at the $B$ factory allows us to test the Standard Model precisely and search for new physics using $B$ mesons, $\tau$ particles, and more.

We have started physics data taking with the full detector system in 2019, and we achieved the world's highest peak luminosity of $3.1 \times 10^{34} \mathrm{~cm}^{-2} \mathrm{~s}^{-1}$ in June 2021 . The recorded integrated luminosity reached $213 \mathrm{fb}^{-1}$ by July 2021 , and will increase to $50 \mathrm{ab}^{-1}$.

The particle identification systems have been fully upgraded for the Belle II experiment. The Time-Of-Propagation (TOP) detector was installed in the barrel region of the Belle II detector.

\section{The Time-of-Propagation (TOP) Detector}

The TOP detector is a ring imaging Cherenkov (RICH) detector. One module of the TOP detector is composed of a plate of quartz radiator and 32 photosensors on the end of the quart plate (Fig. 1a). Sixteen modules surround Central Drift Chamber at $1.2 \mathrm{~m}$ from the beam axis. When a charged particle goes through the quartz radiator, it emits Cherenkov light with a Cherenkov angle $\theta_{C}$, which depends on the particle mass and momentum. Those photons propagate inside the quartz plate with total internal reflection (Fig. 1b), and 20-40 photons are typically detected. The particle type is identified by comparing the temporal and spatial hit patterns of photons with probability density functions (Fig. 1c). High detection efficiency and timing accuracy better than 100 ps are required for a good $K / \pi$ separation as the performance of particle identification is proportional to $\sqrt{N_{\text {photons }}} / \sigma_{t}$, where $N_{\text {photons }}$ is the number of detected photons and $\sigma_{t}$ is the time resolution for a single photon. 


\subsection{Micro-Channel-Plate PMTs (MCP-PMTs) and readout system}

To achieve the high time resolution in the TOP detector, we utilize squared-shape multi-anode Micro-Channel-Plate photomultiplier tubes (MCP-PMTs), which can work in the $1.5 \mathrm{~T}$ magnetic field at the Belle II detector. The photoelectrons are multiplied inside the $10 \mu \mathrm{m}$ diameter channels of the two $400 \mu \mathrm{m}$ thick MCPs. The multiplication in the short path makes the transit time spread quite small and results in $34 \mathrm{ps}$ time resolution for single photon detection with the MCP-PMTs. The MCP-PMTs have a mean quantum efficiency (QE) of $29.3 \%$ at the peak wavelength [1]. The gain is set at $3.0 \times 10^{5}$ by adjusting the applied voltage to keep the threshold efficiency of the readout system above $90 \%$.

The readout system for the TOP detector records waveforms with 2.7 GSample/s on 8192 channels from 512 MCP-PMTs. A board stack consists of four Carrier boards with customdesigned eight-channel waveform sampling ASICs, "Ice Radio Sampler version X" (IRSX), and a Standard Control Read-Out Data (SCROD) board to process photon hits from a quarter of one TOP module. The IRSX ASIC chips store waveforms in an $11 \mu$ s-long analog ring buffer with switching capacitor arrays, and the SCROD board performs feature extraction of the waveform and transfer those data to the downstream of the Belle II data acquisition system. This readout system achieves the average time resolution of $27.6 \mathrm{ps}$, measured with calibration pulses [2].

\subsection{Lifetime of the MCP-PMTs}

The QE degradation of the MCP-PMT is a concern at the Belle II experiment at high rates under harsh beam backgrounds. The degradation is described by the following quadratic function of accumulated output charge $\Sigma_{Q}$ [3],

$$
R_{\mathrm{QE}}=1-0.2\left(\frac{\Sigma_{Q}}{\tau_{\mathrm{QE}}}\right)^{2},
$$

where $R_{\mathrm{QE}}$ is relative $\mathrm{QE}$ from the initial value and $\tau_{\mathrm{QE}}$ is lifetime.

We have installed three types of MCP-PMTs, the conventional, ALD, and life-extended ALD types, in the TOP detector. The lifetime was extended by applying atomic layer deposition (ALD) on the surface of the microchannel plate and reducing residual gas in the tube. The average lifetime was measured to be 1.1 and $10.4 \mathrm{C} / \mathrm{cm}^{2}$ for the Conventional and ALD type samples in a test bench, respectively. The life-extended ALD type is estimated to have a lifetime at least $13.6 \mathrm{C} / \mathrm{cm}^{2}$. The accumulated output charge of the Conventional and ALD types is expected to exceed their lifetime before accumulating $50 \mathrm{ab}^{-1}$. Therefore, we plan to replace the two types with the life-extended ALD type in 2022 and 2026, respectively.

\section{Detector Operation}

\subsection{The MCP-PMTs}

The relative QE above $80 \%$ until the planned replacement of the MCP-PMTs is required to keep the particle identification performance. We set limits on the hit rate of the MCP-PMTs, which is dominated by background hits. Eventually, the TOP detector did not limit anything so far. The limit was set at 3.0 MHz/PMT plus the luminosity-dependent hit rate by the background from the 


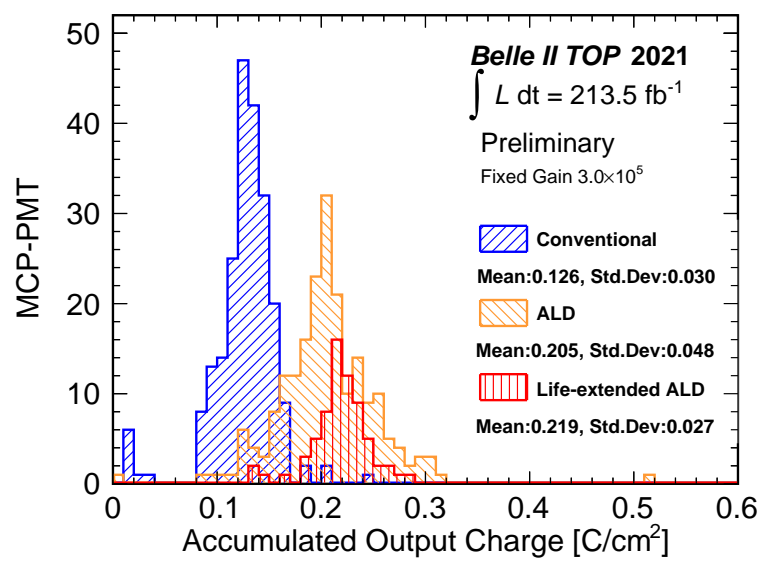

Figure 2: Accumulated output charges of MCP-PMTs by July of 2021 calculated with the nominal gain of $3.0 \times 10^{5}$ for the life-extended ALD type (red), the ALD type (orange), and the conventional type (blue).

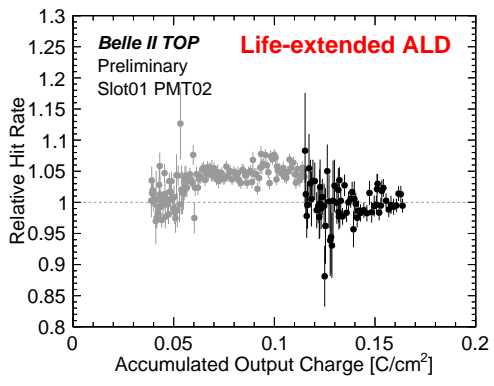

(a)

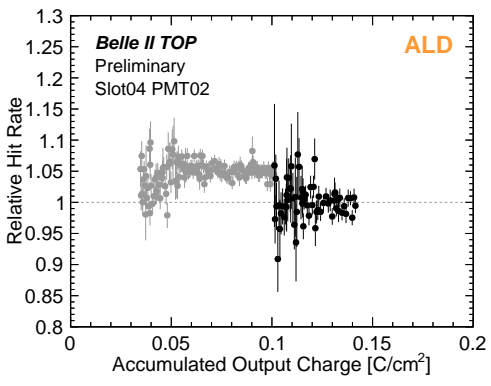

(b)

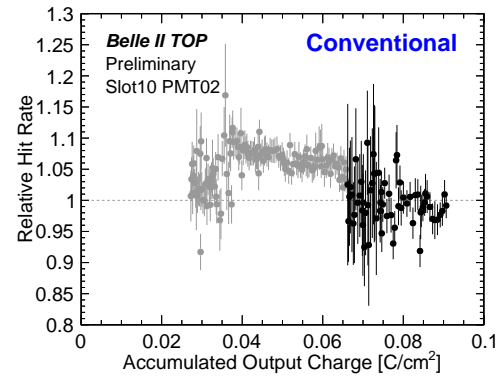

(c)

Figure 3: Typical examples of the hit rate variations. (a) the life-extended ALD, (b) the ALD, and (c) the conventional type. The gray and black points are data with low and high $\mu$ track purity, respectively.

beam collisions during the run period by July 2021. We monitored both accumulated output charge and relative detection efficiency in physics runs. The limit has to be revisited based on the actual QE degradation.

The accumulated output charges are still low for all MCP-PMT types compared to their lifetimes (Fig. 2). The decrease of QE is estimated to be $0.3 \%$ at the average output charge of the conventional type, $0.13 \mathrm{C} / \mathrm{cm}^{2}$, when we assume the lifetime is $1.1 \mathrm{C} / \mathrm{cm}^{2}$.

The relative detection efficiency is evaluated from the number of Cherenkov photons per $\mu$ track in $e^{+} e^{-} \rightarrow \mu^{+} \mu^{-}$events. The relative number of the Cherenkov photons (relative hit rate) has been monitored with the accumulated output charge for each MCP-PMT since November 2019. The data up to April 2021 are shown in Fig. 3. At this moment, the data available for this analysis are $\mu^{+} \mu^{-}$events with different $\mu$ track selections before and after October 2020. The $\mu$ track purity is low before that and is high afterward. Almost all MCP-PMTs have a nearly constant hit rate from October 2020 to April 2021. About 5\% of all the MCP-PMTs have decreased the hit rate. We observed systematic shifts common to all types in the low $\mu$ track purity data even after a correction of the threshold efficiency variation of the readout system. To examine these shifts and validate the 


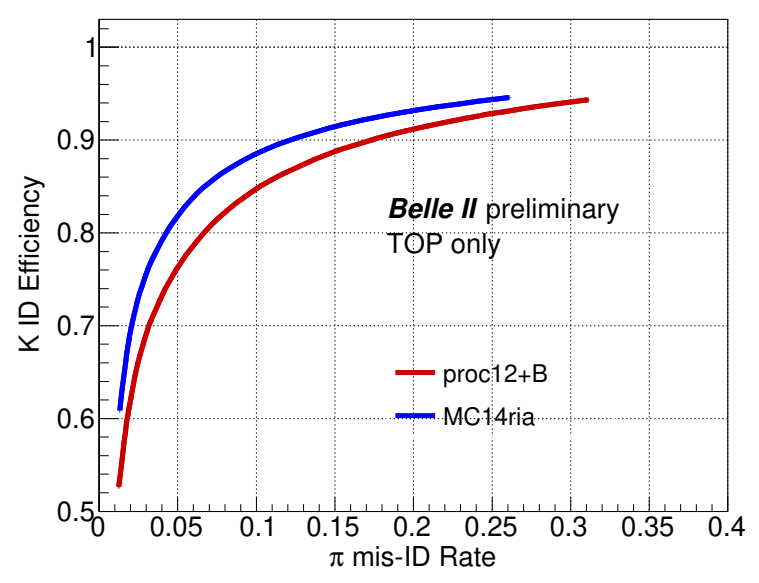

Figure 4: $K$ efficiencies against $\pi$ misidentification rates for the $K$ and $\pi$ entering the TOP detector in the data (red) and the simulation (blue).

evaluation method, we plan to reevaluate the hit rate with the same $\mu$ track selection of high purity for all the run period and plan to evaluate the $\mathrm{QE}$ variation in an independent way using calibration laser runs.

According to our extrapolation of the beam background with the beam current based on background studies, we can store up to $1.5 \mathrm{~A}$ in the positron ring with the new limit of 4.5 MHz/PMT while the maximum current was $860 \mathrm{~mA}$ by July 2021. The limit shall be revisited, in the case of a background increase for a longer beam lifetime by opening beam collimator apertures, or background mitigation by some countermeasures, and delay of the MCP-PMT replacement.

\subsection{The readout system}

One of the main errors during the data taking is a radiation-induced Single Event Upset of the FPGAs on the board stacks. It happened a few times a day. When it happened, we masked the board stack temporarily to immediately restart the data taking. The masked ones were recovered by a power cycle of the board stacks during a beam abort or other downtime.

To realize quick recovery from those errors, we developed tools to detect and fix the errors via GUI semi-automatically. Thanks to these tools, we could resume the data taking in a few minutes in the case of the board stack error and could recover the masked board stacks in ten minutes or less. From February to June 2021, 94.4\% of the all 8192 readout channels were functional. Two board stack, which correspond to $3.1 \%$ of all the channels, were disabled all the time due to a persistent issue. Another board stack was down due to a power supply trouble from the end of May.

\section{Particle Identification Performance}

We evaluated the $K / \pi$ identification performance of the TOP detector using kinematically tagged $K$ and $\pi$ in the decay of $D^{*+} \rightarrow D^{0}\left[\rightarrow K^{-} \pi^{+}\right] \pi_{\text {slow }}^{+}$. The data of $50.8 \mathrm{fb}^{-1}$ collected by April 2021 were used. The TOP detector provides $85 \% K$ identification efficiency at $10 \% \pi$ misidentification rate (Fig. 4). We observed a discrepancy between the data and the Monte Carlo simulation. The discrepancy varies along with the particle incidence position on the quartz bar. A 
study of the number of Cherenkov photons dependent on the incident regions is being performed to understand the discrepancy. The effect of the masked channels on the performance will be confirmed with a more realistic simulation reflecting the number of active channels in the physics runs.

For further performance improvement, identification of the beam bunch timing was added in the reconstruction software, which resulted in a more accurate determination of the hit timing. Moreover, we are developing a waveform template fit to obtain a better time resolution and discrimination power between signal and noise, especially for signals of a small amplitude, as well as new identification methods using neural networks.

\section{Conclusions}

The TOP detector is an upgraded particle identification system for the SuperKEKB/Belle II experiment. The MCP-PMT is a key for the TOP detector, but its lifetime is a concern in the harsh background from beams at high currents. The accumulated charge was at $0.13 \mathrm{C} / \mathrm{cm}^{2}$ on average for the Conventional type, which is about $10 \%$ of the average lifetime, and the relative hit rate per $\mu$ track was nearly constant from October 2020 to April 2021. The fraction of the operational channels was kept at $94.4 \%$ on average in the spring run of 2021 owing to the recovery tools. The TOP detector offers $85 \% \mathrm{~K}$ efficiency at $10 \% \pi$ misidentification rate in the data at the early stage of the Belle II experiment.

\section{References}

[1] K. Matsuoka, Performance of the mcp-pmts of the top counter in the first beam operation of the belle ii experiment, in Proceedings of the 5th International Workshop on New Photon-Detectors (PD18) DOI [https://journals.jps.jp/doi/pdf/10.7566/JPSCP.27.011020].

[2] D. Kotchetkov, O. Hartbrich, M. Andrew, M. Barrett, M. Bessner, V. Bhardwaj et al., Front-end electronic readout system for the belle ii imaging time-of-propagation detector, Nuclear Instruments and Methods in Physics Research Section A: Accelerators, Spectrometers, Detectors and Associated Equipment 941 (2019) 162342.

[3] K. Matsuoka, S. Hirose, T. Iijima, K. Inami, Y. Kato, K. Kobayashi et al., Extension of the mcp-pmt lifetime, Nuclear Instruments and Methods in Physics Research Section A: Accelerators, Spectrometers, Detectors and Associated Equipment 876 (2017) 93. 